\title{
Cognitive Function Characteristics of Parkinson's Disease with Sleep Disorders
}

\author{
Jing Huang, ${ }^{1}$ Wenyan Zhuo, ${ }^{1}$ Yuhu Zhang, ${ }^{2}$ Hongchun Sun, ${ }^{1}$ Huan Chen, ${ }^{1}$ Peipei Zhu, \\ Xiaobo Pan, ${ }^{1}$ Jianhao Yang, ${ }^{1}$ and Lijuan Wang ${ }^{2}$ \\ ${ }^{1}$ Department of Neurology, Zhuhai People's Hospital, Zhuhai 519000, China \\ ${ }^{2}$ Department of Neurology, Guangdong Neuroscience Institute, Guangdong General Hospital and Guangdong Academy of \\ Medical Sciences, Guangzhou 510080, China
}

Correspondence should be addressed to Lijuan Wang; wljgd68@163.com

Received 26 November 2016; Revised 25 March 2017; Accepted 4 April 2017; Published 18 April 2017

Academic Editor: Hélio Teive

Copyright (C) 2017 Jing Huang et al. This is an open access article distributed under the Creative Commons Attribution License, which permits unrestricted use, distribution, and reproduction in any medium, provided the original work is properly cited.

\begin{abstract}
Objective. The aim of this study was to investigate the cognitive function characteristics of Parkinson's disease (PD) with sleep disorders. Methods. Consecutive patients with PD $(n=96)$, patients with primary sleep disorders $(n=76)$, and healthy control subjects $(n=66)$ were assessed. The patients with PD were classified into sleep disorder (PD-SD) and non-sleep disorder (PD-NSD) groups. Results. Among 96 patients with PD, 69 were diagnosed with a sleep disorder. There were 38 sleep disorder cases, 31 RBD cases, and 27 NSD cases. On the Mini-Mental State Examination (MMSE), Montreal Cognitive Assessment (MoCA), and MoCA subtests, patients in the PD-SD, primary sleep disorder, and PD-NSD groups exhibited lower scores than those in the control group. Moreover, the PD-SD patients exhibited more significant cognitive impairment than was observed in the primary sleep disorder patients. In the PD-SD subgroup, the attention scores on the MoCA and on MoCA subtests were lower in the PD with RBD group than in the PD with insomnia group. Conclusion. PD with sleep disorders may exacerbate cognitive dysfunction in patients. PD associated with different types of sleep disorders differentially affects cognitive functions, and patients with PD with RBD exhibited poorer cognitive function than was seen in patients with PD with insomnia.
\end{abstract}

\section{Introduction}

Parkinson's disease (PD) is a typical movement disorder. In addition to motor symptoms, it is characterized by its nonmotor symptoms, including sleep disorders, cognitive dysfunction, autonomic dysfunction, and mental disorders, which seriously affect the quality of life of PD patients [1]. The most common nonmotor symptoms include sleep disorders and cognitive dysfunction. Sleep disorders affect up to $98 \%$ of PD patients [2]. At the time of PD diagnosis, $30-50 \%$ of patients may have mild cognitive impairment (MCI), but they usually do not show dementia unless the patient is diagnosed in the advanced stage of PD [3], and $60-80 \%$ of PD cases develop complete dementia within 10 years $[4,5]$. Sleep quality is strongly correlated with health-related quality of life [6]. Sleep disorders include insomnia, sleep fragmentation, rapid eye movement sleep behavior disorder (RBD), sleep apnea syndrome, restless legs syndrome, and nocturnal enuresis [7]. In $\mathrm{PD}$ patients, insomnia and $\mathrm{RBD}$, particularly the latter, are the most common and widely studied sleep disorders. RBD is not only a sleep disorder but also the preclinical manifestation of several neurodegenerative diseases (such as PD, multiple system atrophy, and Lewy body dementia) [8]. RBD includes a series of clinical symptoms (e.g., screaming, cursing and waving limbs, kicking, and falling out of bed). It accelerates the disease progression and affects the quality of sleep in PD patients, and it may lead to harm or death to patients or bedfellows [9].

The Braak pathological grade hypothesis drew attention to the fact that sleep disorders appear prior to cognitive impairment; moreover, there is a mutual influence between sleep disorders and cognitive impairment. It has been shown that chronic insomnia is an independent risk factor for cognitive impairment in nondepressed older men, whereas 
occasional insomnia does not increase the risk of cognitive impairment [10]. Similar to other neurodegenerative diseases (e.g., Alzheimer's disease), significant sleep problems occur in $\mathrm{PD}$ in combination with significant cognitive dysfunction [11]. Postuma et al. performed a 4-year follow-up study of nondementia PD patients. In their study, 27 cases of PD were associated with RBD, and the incidence of dementia in PD patients with RBD was 15\% after 2 years, $29 \%$ after 3 years, and $48 \%$ after 4 years. In contrast, none of the $15 \mathrm{PD}$ patients who did not present with RBD developed dementia [12]. Sleep disorders may represent an independent risk factor for cognitive impairment; however, most recent studies have only focused on sleep or cognitive conditions for PD patients. The relationship between cognitive function and PD with sleep disorders remains unclear, as do the effects of different types of sleep disorders on cognitive function.

Therefore, this study focused on PD with sleep disorders and comprehensively assessed the sleep quality and cognitive function in PD patients. We classified PD patients according to their sleep disorder types and analyzed the cognitive function characteristics of each subgroup to better characterize the relationship between PD with sleep disorders and cognitive function. Our study provides a novel theoretical basis and clinical application for the treatment, prevention, and early intervention of cognitive impairment in PD patients.

\section{Materials and Methods}

\subsection{Study Population}

2.1.1. General Information. PD patients admitted to the Neurology Department of Guangdong General Hospital and Zhuhai People's Hospital from April 2013 to May 2016 were enrolled in our study. We also assessed a primary sleep disorder group and a healthy control group with the same age, sex distribution, and education level as the PD patients. All subjects were examined by experienced neurologists; they met the United Kingdom PD Society Brain Bank criteria [13] and did not receive anti-PD medication before the evaluation of sleep and the cognition scale. Exclusion criteria were as follows: (1) excessive daytime sleepiness (EDS), sleep attacks, sleep apnea syndrome, restless legs syndrome, parasomnia, and other types of sleep disorders; (2) other serious or unstable physical conditions that affect sleep and cognitive function assessment; (3) pain disorders, drug or alcohol addicts with severe physical illness, or sleep problems being directly a result of physical or mental effects; (4) severe anxiety or depression history and other mental disorders; (5) active epilepsy; (6) history of acute cerebrovascular disease in the previous 3 months; (7) use of sedative or hypnotic drugs within 2 weeks prior to enrollment; (8) presence of aphasia, delirium, or consciousness disorders that affect sleep and cognitive function assessment.

2.1.2. Population Classification. According to the diagnostic criteria of sleep disorders, we classified the PD patients into 2 subgroups, the PD with sleep disorders (PD-SD) group and the PD with no sleep disorders (PD-NSD) group. The $\mathrm{PD}-\mathrm{SD}$ group included the PD with insomnia group and the PD with RBD group [14], whereas the primary sleep disorder group was divided into the primary insomnia group and the primary RBD group. All patients with primary insomnia met the American Psychiatric Association Mental Disorders Diagnostic and Statistical Manual IV diagnostic criteria for primary sleep disorders [15], whereas all primary RBD patients fulfilled the International Sleep Disorders Classification II diagnostic criteria for RBD [16].

\subsection{Study Methods}

2.2.1. Survey Demographics. We administered demographic surveys to all PD patients and to the control group, thereby obtaining general information regarding age, sex, height, weight, and education level and potential associations with other diseases (e.g., recent infection, high blood pressure, diabetes, cancer, and surgical history), age of onset, duration, drug treatment, family history, and concomitant symptoms.

\subsubsection{Clinical Assessments}

(a) Motor Function Assessment. The motor function was assessed using the Unified PD Rating Scale Part III (UPDRSIII), and the disease severity was assessed using the HoehnYahr (H-Y) grade.

(b) Daily Capacity Assessment. Activities of Daily Living Scale (ADL) was used.

(c) Anxiety and Depression Assessment. The Hamilton Anxiety Scale (HAMA) and the Hamilton Depression Scale (HAMD) were used.

(d) Sleep Assessment. The PDSS-2, PSQI, and ESS scales were administered to assess the quality of sleep.

(e) Cognitive Function Assessment. The Mini-Mental State Examination [MMSE] and Montreal Cognitive Assessment [MoCA] were used.

2.3. Statistical Methods. SPSS 13.0 statistical software was used for the statistical analysis. Measurement data are presented as the mean \pm standard deviation $( \pm s d)$. The Kolmogorov-Smirnov test method was adopted for the normality test. For two samples that exhibited a normal distribution, the means were compared using the two independent samples' $t$-test. In contrast, the samples noncompliant with a normal distribution were tested using the Mann-Whitney $U$ test. When multiple samples obeyed the normal distribution and multiple sample averages were compared, one-way ANOVA (analysis of variance) was implemented. Levene's test for equality of variance and Welch's $t$-test for unequal variance were also utilized. The homogeneity of variance was analyzed by the LSD multiple comparison method, and unequal variance was evaluated by Dunnett's T3 multiple comparisons. Comparisons of multiple samples that were not normally distributed were performed using the nonparametric Kruskal-Wallis $H$ test with count data tested using a chisquare test. When $P<0.05$, we considered the difference to be statistically significant. 
TABLE 1: Comparison of subjective sleep in PD-SD and PD-NSD.

\begin{tabular}{lcccc}
\hline & PD-SD (69) & PD-NSD (27) & $Z$ & $P$ value \\
\hline PDSS-2-T & $20.69 \pm 10.54$ & $10.00 \pm 5.90$ & -4.130 & 0.000 \\
ESS-T & $6.21 \pm 4.39$ & $4.39 \pm 3.65$ & -1.496 & 0.135 \\
PSQI-T & $9.08 \pm 4.22$ & $6.44 \pm 3.88$ & -2.329 & 0.020 \\
\hline
\end{tabular}

TABLE 2: Comparison between PD-NSD and PD with sleep disorders.

\begin{tabular}{|c|c|c|c|c|}
\hline & PD-SD (69) & PD-NSD (27) & $t / Z / \chi^{2}$ & $P$ value \\
\hline Age, y & $61.27 \pm 11.38$ & $56.28 \pm 13.81$ & 1.496 & 0.139 \\
\hline Gender, M/F (\%) & $32 / 37$ & $11 / 16$ & 0.091 & 0.763 \\
\hline Education, y & $10.27 \pm 3.92$ & $12.39 \pm 3.79$ & -1.805 & 0.071 \\
\hline Age of onset, $y$ & $57.25 \pm 11.24$ & $53.06 \pm 12.87$ & 1.298 & 0.199 \\
\hline PD duration, $y$ & $3.52 \pm 2.96$ & $3.31 \pm 4.48$ & -1.042 & 0.297 \\
\hline UPDRS-III & $28.23 \pm 10.10$ & $24.50 \pm 10.84$ & -2.017 & 0.067 \\
\hline Hoehn-Yahr scale & $2.68 \pm 4.11$ & $1.81 \pm 0.94$ & -1.868 & 0.062 \\
\hline HAMA & $9.33 \pm 6.77$ & $7.11 \pm 3.61$ & -0.846 & 0.397 \\
\hline HAMD & $9.69 \pm 7.33$ & $9.39 \pm 6.09$ & -0.253 & 0.800 \\
\hline $\mathrm{ADL}$ & $20.23 \pm 9.87$ & $19.83 \pm 8.42$ & -0.610 & 0.701 \\
\hline
\end{tabular}

TABLE 3: Cognitive function characteristic analysis comparing the PD-SD, PD-NSD, primary sleep disorder, and normal control groups.

\begin{tabular}{|c|c|c|c|c|c|c|}
\hline & PD-SD (69) & PD-NSD (27) & Primary sleep disorders (76) & Normal controls (66) & $\chi^{2}$ & $P$ value \\
\hline MMSE & $25.48 \pm 3.82$ & $28.44 \pm 1.79$ & $27.15 \pm 3.65$ & $28.92 \pm 1.50$ & 25.512 & 0.000 \\
\hline $\mathrm{MoCA}$ & $19.00 \pm 4.99$ & $25.50 \pm 3.20$ & $22.54 \pm 5.75$ & $27.00 \pm 2.49$ & 47.963 & 0.000 \\
\hline Visuospatial and execution & $2.50 \pm 1.38$ & $3.72 \pm 1.02$ & $3.15 \pm 1.71$ & $4.50 \pm 0.83$ & 34.282 & 0.000 \\
\hline Naming & $2.35 \pm 0.67$ & $2.78 \pm 0.55$ & $2.87 \pm 0.54$ & $3.00 \pm 0.00$ & 37.522 & 0.000 \\
\hline Attention & $4.50 \pm 1.35$ & $5.39 \pm 0.92$ & $5.48 \pm 0.91$ & $5.75 \pm 0.44$ & 27.669 & 0.000 \\
\hline Language & $2.25 \pm 0.91$ & $2.78 \pm 0.55$ & $2.54 \pm 0.59$ & $2.75 \pm 0.53$ & 9.312 & 0.025 \\
\hline Abstract & $0.96 \pm 0.82$ & $1.39 \pm 0.70$ & $1.15 \pm 0.76$ & $1.54 \pm 0.66$ & 10.108 & 0.018 \\
\hline Delayed recall & $1.38 \pm 1.10$ & $3.61 \pm 1.24$ & $1.91 \pm 1.76$ & $3.58 \pm 1.28$ & 41.662 & 0.000 \\
\hline Orientation & $5.08 \pm 1.11$ & $5.83 \pm 0.38$ & $5.70 \pm 0.89$ & $5.92 \pm 0.28$ & 26.226 & 0.000 \\
\hline
\end{tabular}

\section{Results}

3.1. General Information. The $96 \mathrm{PD}$ patients included 41 males and 55 females (mean age $58.91 \pm 12.19$ years); 76 patients were included in the primary sleep disorders group, including 43 males and 33 females (mean age 57.30 \pm 13.36 years). The normal control group consisted of 66 subjects, including 36 males and 30 females (mean age $56.63 \pm 9.84$ years).

According to the diagnostic criteria for PD sleep disorders, the PD patients included a PD-SD group of 69 cases, which accounted for $72.7 \%$ of the total PD patients $(38$ cases in the PD with insomnia group, or $40.1 \%$ of the total PD patients; 31 cases in the PD with RBD group, or 31.8\%). There were 27 cases in the PD-NSD group, accounting for $27.3 \%$ of the total PD patients. The PD-SD and PD-NSD groups showed significant differences in the PDSS-2-T and PSQI$\mathrm{T}$ scores but no significant differences in the ESS scores (Table 1). There were no significant differences in the age, age of onset, duration, years of education, UPDRS-III, HY classification, HAMA score, HAMD score, or ADL score $(P>0.05)$ (Table 2).

3.2. Cognitive Function Analysis in PD with Sleep Disorders. (1) There were significant differences in cognitive function among the PD-SD, PD-NSD, primary sleep disorder, and normal control groups. For the PD-SD and primary sleep disorder groups, the MMSE scores were only $25.48 \pm 3.82$ and $27.15 \pm 3.65$, and the MoCA scores were only $19.00 \pm 4.99$ and $22.54 \pm 5.75$, respectively, when using multiple independent samples. Similarly, for the overall cognitive function (MMSE and MoCA) and MoCA subtest assessments, the PD-SD and primary sleep disorder groups showed significantly lower scores (Table 3). Furthermore, using one-way ANOVA with the LSD test for cases in which Levene's test showed homogeneity of variance and with Dunnett's T3 method for cases of nonhomogeneous variance, we found that the PD$\mathrm{SD}$ group showed more significant impairment of cognitive 
TABLE 4: Cognitive function characteristic analysis comparing the PD-SD, PD-NSD, primary sleep disorders, and normal control groups.

\begin{tabular}{|c|c|c|c|c|c|c|}
\hline & $A$ versus $B$ & A versus $C$ & A versus $\mathrm{D}$ & $\mathrm{B}$ versus $\mathrm{C}$ & $B$ versus $D$ & C versus D \\
\hline MMSE & 0.004 & 0.038 & 0.000 & 0.590 & 0.922 & 0.043 \\
\hline MoCA & 0.000 & 0.001 & 0.000 & 0.196 & 0.414 & 0.000 \\
\hline Visuospatial and execution & 0.012 & 0.044 & 0.000 & 0.890 & 0.066 & 0.000 \\
\hline Naming & 0.021 & 0.000 & 0.000 & 0.910 & 0.383 & 0.403 \\
\hline Attention & 0.042 & 0.000 & 0.000 & 0.998 & 0.715 & 0.791 \\
\hline Language & 0.052 & 0.816 & 0.062 & 0.345 & 1.000 & 0.440 \\
\hline Abstract & 0.261 & 0.843 & 0.014 & 0.812 & 0.972 & 0.163 \\
\hline Delayed recall & 0.000 & 0.427 & 0.000 & 0.000 & 1.000 & 0.000 \\
\hline Orientation & 0.005 & 0.000 & 0.000 & 1.000 & 0.965 & 0.860 \\
\hline
\end{tabular}

A: PD-SD; B: PD-NSD; C: primary sleep disorders; D: normal control.

TABLE 5: Cognitive function characteristic analysis comparing two subtypes of PD-SD.

\begin{tabular}{|c|c|c|c|c|}
\hline & PD with insomnia (38) & PD with RBD (31) & $Z$ & $P$ value \\
\hline MMSE & $26.11 \pm 3.86$ & $24.67 \pm 3.71$ & -1.914 & 0.056 \\
\hline $\mathrm{MoCA}$ & $20.07 \pm 5.36$ & $17.62 \pm 4.20$ & -2.187 & 0.029 \\
\hline Visuospatial and execution & $2.74 \pm 1.32$ & $2.19 \pm 1.44$ & -1.668 & 0.095 \\
\hline Naming & $2.41 \pm 0.69$ & $2.29 \pm 0.64$ & -0.746 & 0.456 \\
\hline Attention & $4.93 \pm 1.27$ & $3.95 \pm 1.28$ & -2.686 & 0.007 \\
\hline Language & $2.26 \pm 0.94$ & $2.24 \pm 0.89$ & 0.161 & 0.872 \\
\hline Abstract & $1.11 \pm 0.80$ & $0.76 \pm 0.83$ & -1.466 & 0.143 \\
\hline Delayed recall & $1.41 \pm 1.25$ & $1.33 \pm 0.91$ & -0.011 & 0.991 \\
\hline Orientation & $5.19 \pm 1.18$ & $4.95 \pm 1.02$ & -1.247 & 0.212 \\
\hline
\end{tabular}

function than did the primary sleep disorder group, based on measures such as the MMSE and MoCA scores and the Naming, Attention, and Orientation subitems in the MoCA (Table 4).

(2) Comparing the subtypes of PD-SD, the MoCA score was lower in the PD with RBD group than in the PD with insomnia group. Similarly, the attention score of the MoCA subtest was lower in the PD with RBD group than in the PD with insomnia group (Table 5).

\section{Discussion}

To date, the combination of PD with sleep disorders and cognitive function has been poorly investigated, particularly regarding the difference between the effects of different types of sleep disorders on cognitive function. Adequate sleep is an important requirement for good memory and efficient executive function. The cognitive changes that occur in early-to-moderate PD are primarily deficits in executive function and memory [17-19], which are the same domains as those affected in individuals with insomnia [20]. Studies have investigated the impact of PD with RBD on cognitive function, but how PD with insomnia affects cognitive function remains poorly understood. To address these gaps, our study compares cognitive functions between PD patients with RBD and PD patients with insomnia. Gagnon et al. determined that the MCI incidence rate was $73 \%$ in $\mathrm{PD}$ patients with RBD but only $50 \%$ in primary $\mathrm{RBD}$ patients. Moreover, in the PD without RBD group, $11 \%$ of patients developed MCI, compared with an $8 \%$ incidence rate in the normal control group [21]. Furthermore, studies have indicated that there was no significant difference in motor function between PD with and without RBD. However, PD patients with RBD performed worse in memory, visuospatial, and executive functions [22]. Postuma et al. determined that $\mathrm{PD}$ patients with RBD were more likely to develop dementia than were PD patients without RBD [12]. Sleep quality in PD is significantly correlated with cognition, and it differentially impacts attention and executive function, thereby furthering our understanding of the link between sleep and cognition [23]. Our results were consistent with previous studies, and there were no significant differences in motor function or daily living ability between the PD with sleep disorder and PD with normal sleep groups. Interestingly, both PD with sleep disorder and primary sleep disorder patients showed significant impairments in the overall cognitive function and MoCA subtests compared with the PD with normal sleep and normal control groups. Moreover, the scores of the PD patients with sleep disorders were significantly lower than those of the primary sleep disorder patients. Thus, patients with PD with sleep disorders have a greater risk of cognitive dysfunction. Clinicians need to solve PD patients' sleep problems as early as possible in order to delay the associated cognitive decline. 
In the analysis of the subtypes of PD with sleep disorders, the total MoCA score was lower in the PD with RBD group than in the PD with insomnia group. Furthermore, for the MoCA subtests, the PD patients with RBD had lower attention scores and more significant cognitive impairment compared with the PD patients with insomnia, particularly regarding attention. Therefore, poor sleepers exhibited worse performance on tests of global cognition. A sleep disorder is considered as an independent risk factor for PD-related cognitive impairment, whereas the impact of RBD on cognitive function is more obvious.

Cognitive function in patients with primary RBD has received increasing attention. $\mathrm{RBD}$ may be associated with preclinical presentation of a number of neurodegenerative diseases (multiple system atrophy, Lewy body dementia, and Parkinson's disease). The cognitive function of PD with $\mathrm{RBD}$ is also currently receiving substantial attention in the study of Parkinson's disease. Primary RBD patients often present with visuospatial impairment, attention dysfunction, executive dysfunction, or verbal memory loss [24]. Studies have indicated that $73 \%$ of PD patients with RBD present with MCI [25]. As previously discussed, RBD is considered an independent risk factor for PD-associated cognitive impairment. Consistent with other research results, our study demonstrated that PD patients with RBD have poorer cognitive function. To date, the pathophysiology underlying the effects of RBD on cognitive function remains unclear. First, the pathological mechanism of RBD may be related to the abnormalities of the tegmentum and the ascending reticular activating system [26]. Second, Braak et al. have suggested that the pathological evolution of PD that involves the medulla oblongata and pontine tegmentum occurs via a twophase pathological process, and these are common regions of RBD occurrence [27]. The damaged regions of the cerebral cortex in PD or RBD patients are believed to regulate neural activity, and nervous system conduction disorders may affect cognitive function. It has been demonstrated that the brain metabolism perfusion patterns differ between PD and PD cognitive impairment patients. In PD cognitive impairment patients, there have been reports of reduced perfusion in the frontal and parietal regions and increased perfusion in the brain stem and cerebellum [28]. This abnormal metabolic perfusion has also been identified in studies of primary RBD [29]. The clinical manifestations of PD are significantly heterogeneous and may be related to the position and degree of neuronal injury. Furthermore, the mechanisms of neurodegeneration may differ between PD patients with and without RBD. However, there are no well-established imaging studies to elucidate the neuropathological mechanisms in PD with RBD; thus, additional studies are required to further illustrate its pathogenesis.

In summary, sleep disorders may exacerbate the occurrence and development of PD. Neurologists should focus on sleep disorders in PD patients and be fully aware of different types of sleep disorders to achieve early diagnosis, early intervention, and early treatment. This knowledge is essential for improving patient quality of life, reducing the risk of cognitive impairment, and slowing the progression of cognitive decline.

\section{Conflicts of Interest}

The authors declare that they have no financial or other conflicts of interest in relation to this research and its publication.

\section{Authors' Contributions}

Jing Huang and Wenyan Zhuo contributed equally to this work.

\section{References}

[1] K. R. Chaudhuri, C. Prieto-Jurcynska, Y. Naidu et al., "The nondeclaration of nonmotor symptoms of Parkinson's disease to health care professionals: An international study using the nonmotor symptoms questionnaire," Movement Disorders, vol. 25, no. 6, pp. 704-709, 2010.

[2] C. H. Adler and M. J. Thorpy, "Sleep issues in Parkinson's disease," Neurology, vol. 64, no. 12, pp. S12-S20, 2005.

[3] D. Aarsland, J. T. Kvaløy, K. Andersen et al., "The effect of age of onset of PD on risk of dementia," Journal of Neurology, vol. 254, no. 1, pp. 38-45, 2007.

[4] D. Aarsland, K. Andersen, J. P. Larsen, A. Lolk, and P. KraghSørensen, "Prevalence and characteristics of dementia in Parkinson disease: An 8-year prospective study," Archives of Neurology, vol. 60, no. 3, pp. 387-392, 2003.

[5] T. C. Buter, A. Van Den Hout, F. E. Matthews, J. P. Larsen, C. Brayne, and D. Aarsland, "Dementia and survival in Parkinson disease: A 12-year population study," Neurology, vol. 70, no. 13, pp. 1017-1022, 2008.

[6] T. Scaravilli, E. Gasparoli, F. Rinaldi, G. Polesello, and F. Bracco, "Health-related quality of life and sleep disorders in Parkinson's disease," Neurological Sciences, vol. 24, no. 3, pp. 209-210, 2003.

[7] E. Havlikova, J. P. V. Dijk, I. Nagyova et al., “The impact of sleep and mood disorders on quality of life in Parkinson's disease patients," Journal of Neurology, vol. 258, no. 12, pp. 2222-2229, 2011.

[8] A. Iranzo, A. Fernández-Arcos, E. Tolosa et al., "Neurodegenerative disorder risk in idiopathic REM sleep behavior disorder: Study in 174 patients," PLoS ONE, vol. 9, no. 2, Article ID e89741, 2014.

[9] C. H. Schenck, S. A. Lee, M. A. C. Bornemann, and M. W. Mahowald, "Potentially lethal behaviors associated with rapid eye movement sleep behavior disorder: Review of the literature and forensic implications," Journal of Forensic Sciences, vol. 54, no. 6, pp. 1475-1484, 2009.

[10] S. Beaulieu-Bonneau and C. Hudon, "Sleep disturbances in older adults with mild cognitive impairment," International Psychogeriatrics, vol. 21, no. 4, pp. 654-666, 2009.

[11] M. V. Vitiello and S. Borson, "Sleep disturbances in patients with alzheimer's disease: Epidemiology, pathophysiology and treatment," CNS Drugs, vol. 15, no. 10, pp. 777-796, 2001.

[12] R. B. Postuma, J.-A. Bertrand, J. Montplaisir et al., "Rapid eye movement sleep behavior disorder and risk of dementia in Parkinson's disease: A prospective study," Movement Disorders, vol. 27, no. 6, pp. 720-726, 2012.

[13] A. J. Hughes, S. E. Daniel, L. Kilford, and A. J. Lees, "Accuracy of clinical diagnosis of idiopathic Parkinsons disease: a clinicopathological study of 100 cases," Journal of Neurology, Neurosurgery \& Psychiatry, vol. 55, no. 3, pp. 181-184, 1992. 
[14] M. Louter, W. C. C. A. Aarden, J. Lion, B. R. Bloem, and S. Overeem, "Recognition and diagnosis of sleep disorders in Parkinson's disease," Journal of Neurology, vol. 259, no. 10, pp. 2031-2040, 2012.

[15] American Psychiatric Association, Diagnostic and Statistical Manual of Mental Disorders: DSM-IV, American Psychiatric Association, Washington, DC, USA, 4th edition, 1994.

[16] American Academy of Sleep Medicine, International Classification of Sleep Disorders Diagnostic and Coding Manual, American Academy of Sleep Medicine, Westchester, Ill, USA, 2nd edition, 2005.

[17] A. E. Taylor, J. A. Saint-Cyr, and A. E. Lang, "Memory and learning in early Parkinson's disease: Evidence for a "frontal lobe syndrome"," Brain and Cognition, vol. 13, no. 2, pp. 211-232, 1990.

[18] A. M. Owen, M. James, P. N. Leigh et al., "Fronto-striatal cognitive deficits at different stages of parkinson's disease," Brain, vol. 115, no. 6, pp. 1727-1751, 1992.

[19] D. Muslimović, B. Post, J. D. Speelman, and B. Schmand, "Cognitive profile of patients with newly diagnosed Parkinson disease," Neurology, vol. 65, no. 8, pp. 1239-1245, 2005.

[20] É. Fortier-Brochu, S. Beaulieu-Bonneau, H. Ivers, and C. M. Morin, "Insomnia and daytime cognitive performance: A metaanalysis," Sleep Medicine Reviews, vol. 16, no. 1, pp. 83-94, 2012.

[21] J.-F. Gagnon, M. Vendette, R. B. Postuma et al., "Mild cognitive impairment in rapid eye movement sleep behavior disorder and Parkinson's disease," Annals of Neurology, vol. 66, no. 1, pp. 3947, 2009.

[22] J.-R. Zhang, J. Chen, Z.-J. Yang et al., "Rapid eye movement sleep behavior disorder symptoms correlate with domains of cognitive impairment in parkinson's disease," Chinese Medical Journal, vol. 129, no. 4, pp. 379-385, 2016.

[23] K. Stavitsky, S. Neargarder, Y. Bogdanova, P. McNamara, and A. Cronin-Golomb, "The impact of sleep quality on cognitive functioning in Parkinson's disease," Journal of the International Neuropsychological Society, vol. 18, no. 1, pp. 108-117, 2012.

[24] M. L. Fantini, E. Farini, P. Ortelli et al., "Longitudinal study of cognitive function in idiopathic REM sleep behavior disorder," Sleep, vol. 34, no. 5, pp. 619-625, 2011.

[25] I. Djonlagic, M. Guo, P. Matteis, A. Carusona, R. Stickgold, and A. Malhotra, "Untreated sleep-disordered breathing: Links to aging-related decline in sleep-dependent memory consolidation," PLoS ONE, vol. 9, no. 1, Article ID e85918, 2014.

[26] J.-F. Gagnon, R. B. Postuma, S. Mazza, J. Doyon, and J. Montplaisir, "Rapid-eye-movement sleep behaviour disorder and neurodegenerative diseases," Lancet Neurology, vol. 5, no. 5, pp. 424-432, 2006.

[27] H. Braak, K. Del Tredici, U. Rüb, R. A. I. De Vos, E. N. H. Jansen Steur, and E. Braak, "Staging of brain pathology related to sporadic Parkinson's disease," Neurobiology of Aging, vol. 24, no. 2, pp. 197-211, 2003.

[28] C. Huang, P. Mattis, K. Perrine, N. Brown, V. Dhawan, and D. Eidelberg, "Metabolic abnormalities associated with mild cognitive impairment in Parkinson disease," Neurology, vol. 70, no. 16, pp. 1470-1477, 2008.

[29] S. Mazza, J. P. Soucy, P. Gravel et al., "Assessing whole brain perfusion changes in patients with REM sleep behavior disorder," Neurology, vol. 67, no. 9, pp. 1618-1622, 2006. 


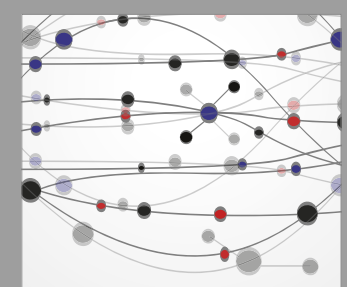

The Scientific World Journal
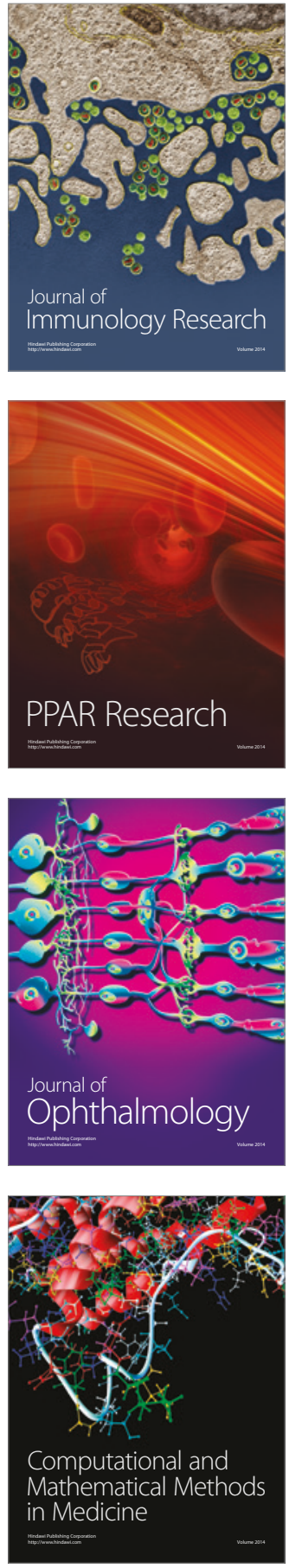

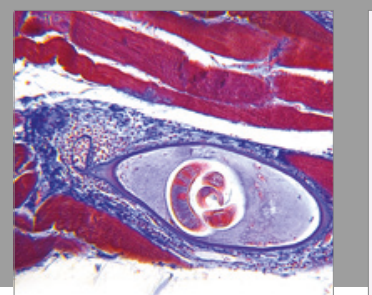

Gastroenterology Research and Practice
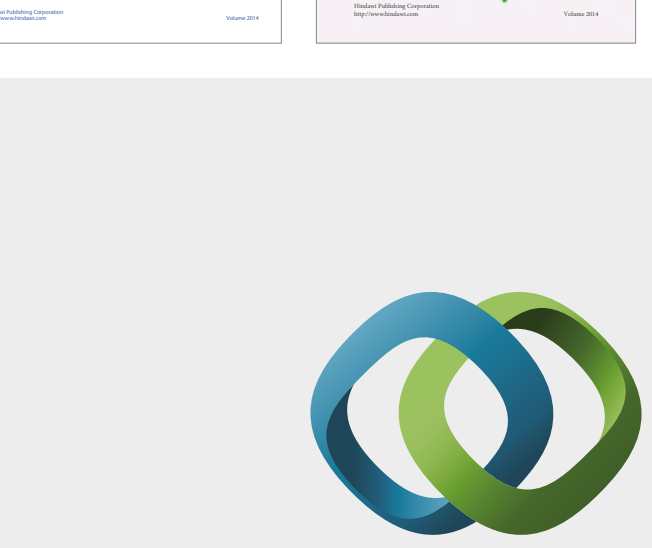

\section{Hindawi}

Submit your manuscripts at

https://www.hindawi.com
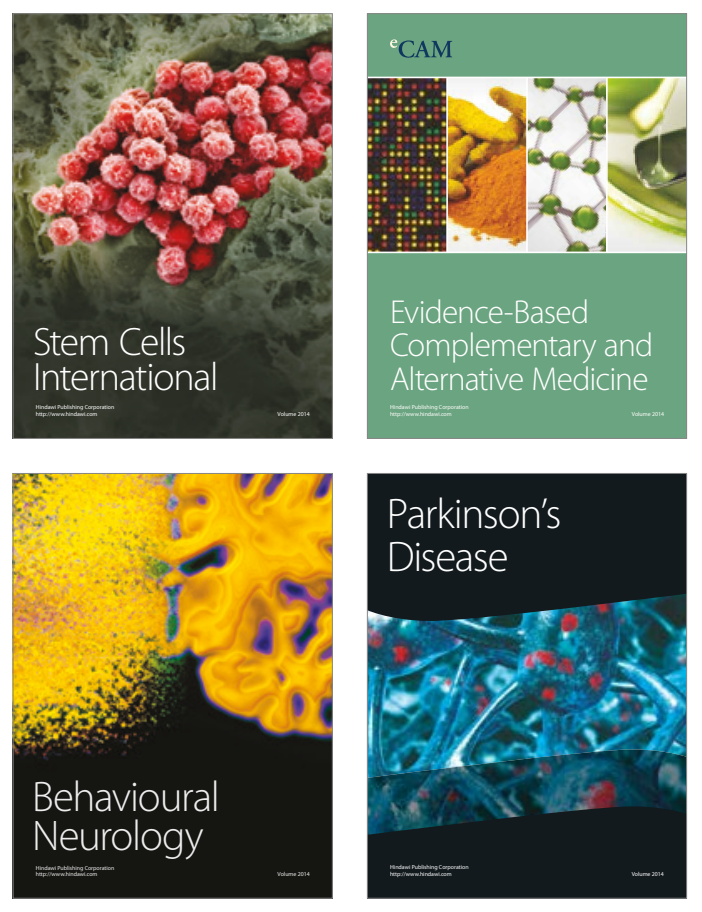
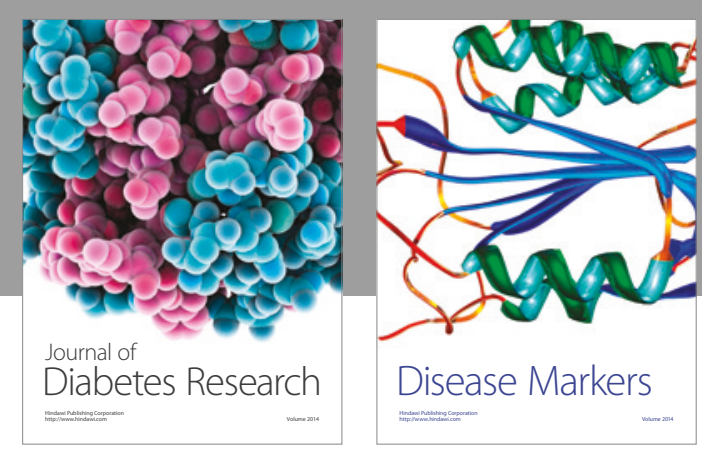

Disease Markers
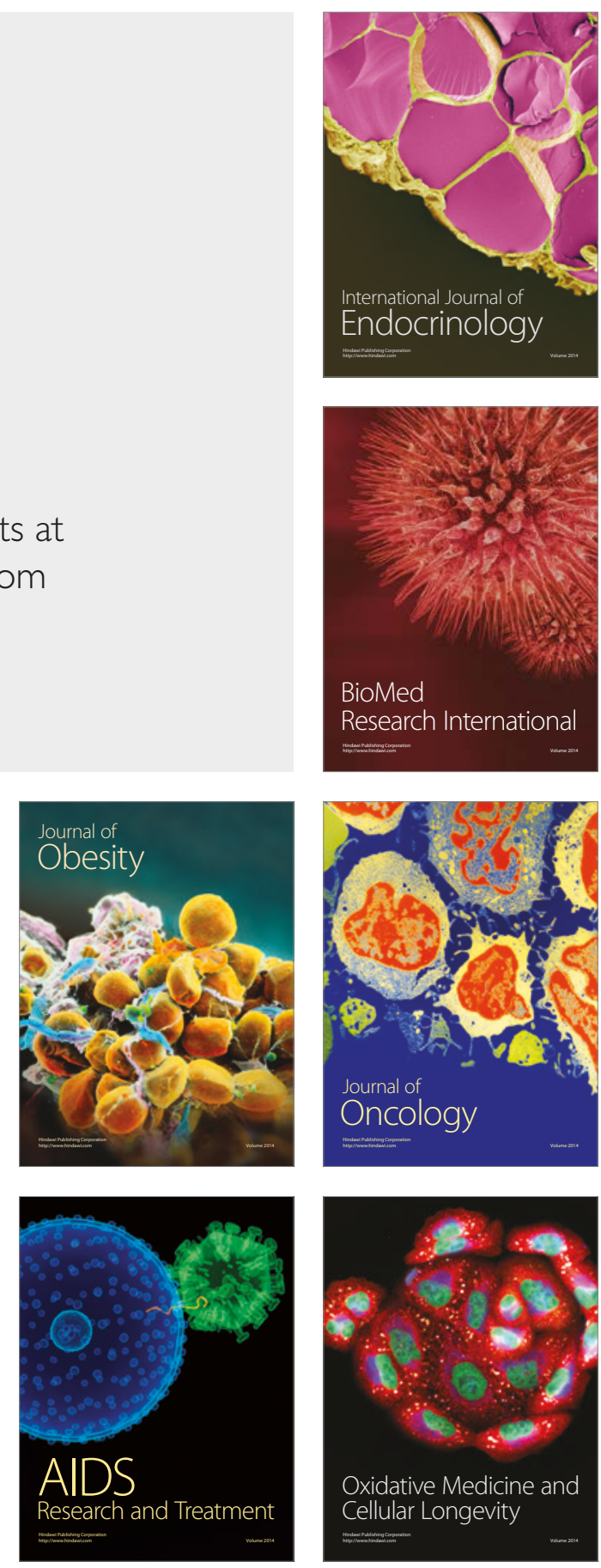